\title{
To Trade, Or Not To Trade: A Model of Regret and Investment"
}

\author{
Jie Qin $^{\mathrm{a}}$
}

APEA, Seattle

March 17, 2012

\begin{abstract}
This paper examines the effect of regret on investment in a market where investors can choose to trade, or not to trade, an asset with uncertain value. Investors face the tradeoff between the expect utility of investment return and the expected disutility of regret. When the movement of price is moderate, investors take positions according to their private information. When price becomes more volatile, some investors will ignore their private information and exit the market. In case the price is extremely high or extremely low, regret has such a strong effect that a market-wide cascade will come into occurrence.
\end{abstract}

JEL classification: G11; D03; D82

Keywords: Regret, Investment, Trading, Cascade, Herd

\footnotetext{
* The author is grateful for comments and suggestions of Keiichi Hori, Yoshihiro Tsutsui and seminar participants at Osaka University and Ritsumeikan University. Financial support from the Ishii Memorial Securities Research Promotion Foundation is gratefully acknowledged.

a Professor, Department of Economics, Ritsumeikan University. Email: khata@ec.ritsumei.ac.jp
} 


\title{
To Trade, Or Not To Trade: A Model of Regret and Investment
}

\author{
Jie Qin \\ "I want to live my life so that my nights are full of regrets." \\ F. Scott Fitzgerald \\ "I want to live my life so that my nights are not full of regrets." \\ D. H. Lawrence
}

\section{Introduction}

In literature and adages, regret is often described as the feeling with the worst taste; in the real world where life is an endless flow of choices, everybody knows the taste the regret, and will experience it again and again. Empirical and experimental researches of gamble and consumer behavior, as reviewed by Zeenlenberg (1999) and Zeelenberg et al. (2000), suggest that after repeated experience of regret, individuals tend to exhibit regret aversion which has a profound effect on decision making. ${ }^{1}$ Researches of neuroscience, for example, Camille et al. (2004) and Coricelli et al. (2005), find that regret and its avoidance is particularly related with the activity of the orbitofrontal cortex of brain. ${ }^{2}$

In economics, "regret theory" is developed by Bell $(1982,1983)$ and Loomes and Sugden (1982, 1987) as an alternative to the standard theory of expected utility. To analyze regret averse individual's decision-making problem under uncertainty, they propose the following "modified utility function":

$$
\begin{aligned}
& u\left(r_{i}, r_{j}\right)=r_{i}+f\left(r_{i}-r_{j}\right) \\
& u\left(r_{j}, r_{j}\right)=r_{j}+f\left(r_{j}-r_{i}\right)
\end{aligned}
$$

where $r_{i}$ is the uncertain return on asset- $i, r_{j}$ the return on asset- $j$, and $f(\cdot)$ is a strictly increasing and decreasingly concave function with $f(0)=0$. For an individual who has chosen asset- $i$, her utility is increasing in $r_{i}$, the actual return on asset- $i$, and is decreasing in $r_{j}$, the return she would obtain if she had chosen asset- $j$. The function $f\left(r_{i}-r_{j}\right)$ measures the individual's regret on her decision, and is called "regret-rejoice function" by Loomes and Sugden (1982). Under uncertainty about asset returns, the necessary and sufficient condition for the individual to choose asset- $i$ is

$$
\mathrm{E}\left[u\left(r_{i}, r_{j}\right)\right]>E\left[u\left(r_{j}, r_{i}\right)\right]
$$

\footnotetext{
${ }^{1}$ Disappointment is a psychological effect that is similar to regret, with both come from counterfactual thinking. However, regret is related to choices while disappointment is situation-focused. For more discussions about the deference between regret and disappointment, see Bell (1985), Loomes and Sugden (1986), and Zeelenberg et al. (1998).

${ }^{2}$ For a survey of theoretical and empirical researches of regret, see Gilovich and Medvec (1995).
} 
With this modified utility function, Bell (1982) and Loomes and Sugden (1982) show that they can explain the paradox in Allais (1953) and the experimental evidence in Kahneman and Tversky's (1979)

Quiggin (1994) extends the pairwise utility function of regret theory into multiple-choices:

$$
u\left(r_{i}\right)=r_{i}+f\left(r_{i}-\max \left\{r_{i}\right\}\right)
$$

where $\max \left\{r_{i}\right\}$ is the best possible result among all choices. Because $r_{i}-\max \left\{r_{i}\right\} \leq 0$, there is always a tradeoff between the actual return on the chosen asset and the regret that the individual feels when she compares the actual return with the best possible result.

Recently, regret theory is also applied to the study of financial markets; for example, Dodonova (2005) develops an asset pricing model in the existence of regret averse investors, Fogel and Berry (2006) examine the relation between regret aversion and disposition effect, and Michenaud and Solnik (2008) analyze regret averse investor's choices of currency hedging.

In the present paper, we examine the effect of regret on investment in a market where investors can choose to trade, or not to trade, an asset with uncertain value. Following Glosten and Milgrom (1985), we adopt the framework of sequential trading model: in the market there are informed traders, who have private information about the asset, as well as liquidity traders, who randomly submit buy or sell orders; price is set by a risk-neutral market maker, who randomly executes one order at each trading round; public information is updated according to order flow after each trading round.

However, different to Glosten and Milgrom (1985), we assume informed traders are regret averse. Following Bell (1982, 1983), Loomes and Sugden (1982, 1987) and Quiggin (1994), we employ an modified utility function that includes both the utility on investment return and the disutility of regret. Hence, in our model, although informed traders can earn a positive expected return by using their private information, they face the tradeoff between expected return and expected disutility of regret. Moreover, in our model investors can choose not to trade. In the original model of Glosten and Milgrom (1985), investors can only choose between buy and sell; however, in the real world, people not only regret on their actions, but also regret on doing nothing. Hence, the extension in the present paper makes it possible to examine the effect of regret in a setting that is relatively close to the real world.

We first examine the case where investors' attitude toward regret does not depend on their positions in the market; that is, whether they are trading or not, they have same magnitude of regret aversion. In this case, it is found that investors always choose trading. For example, if an investor observes a positive signal about asset value, she will choose to buy as long as the price is higher than a curtain level; if the price drops below this level, she will choose to sell instead of just staying aside. The reason is that if investors do not trade, they will regret this choice after they know the true value of the asset.

We then extend the assumption about regret such that investors' magnitude of regret aversion depends on their positions in the market. Specifically, we are interested in the case where investors' magnitude of regret aversion is relatively small when they do not trade. As shown by Gilovich and Medvec (1995) and many other researches, in long-term, people tend to regret more about doing nothing. For example, when asked what the most regrettable failure in life is, many people choose something they fell to do, such as missed educational opportunity, missed romantic opportunity, and so on. However, In short-term, people tend to regret more on their recent actions. Because trading has relatively short time-horizon compared with decisions of career and marriage, the extended model of the present paper assumes that investors' regret on not trading is smaller than that on a wrong trading position. In this case the following results are obtained. When the market price takes moderate values, investors take positions according to their private information. When asset price rises above a certain level, then, 
while investors with positive signals still buy, those with negative signals will choose not to trade; if price becomes even higher, than all investors will choose to buy in spite of their private information. Symmetrically, when asset price drops, investors with positive signals first choose to stay aside; then, if the price drops even more deeply, they will re-enter the market and join the sellers.

This paper applies regret theory to a standard trading model in finance literature. It shows explicitly the effect of regret on investors' trading strategies. Furthermore, it provides an alternative rational to informational cascades in financial markets. As noted by Bikhchandani et al. (1992), an informational cascade occurs when it is optimal for an individual to follow the behavior of the preceding individual without regard to her own information. ${ }^{3}$ In the model of the present paper, when the price is very low or very high, a "partial cascade" occurs where some informed traders ignore their private information and do not trade. If the price takes even more extreme values, a partial cascade will develop into a full-scale cascade. The reason for investors to join a partial cascade or a full-scale cascade is that they do not want to bet against the market and regret afterwards.

The rest of the paper is organized as follows. Section 2 describes the specification of investors' preference as well as the framework of the market. Section 3 derives investors' trading strategies in the case where they have same level of regret aversion whether they trade or do not trade. Section 4 generalizes the model and analyzes the case where investors have weak regret aversion when they do not trade. Results about partial cascades and full scale cascades are also discussed. Section 5 concludes.

\section{The Model}

In the present paper, we investigate investors' trading strategy in a market where a risky asset is traded by investors who can choose among three positions: long, short, and not trading. The value of the asset, denoted by $V$, is uncertain with $\operatorname{Pr}\{V=1\}=\mu$ and $\operatorname{Pr}\{V=0\}=1-\mu$. Let $x \in\{1,0,-1\}$ denote an investor's choice: $x=1$ if the investor buys one unit of the asset, $x=-1$ if she sells one unit of the asset, and $x=0$ if she decides not to trade. Let $p$ denote the price of the asset and $r=r(x)$ denote the return on position $x$. Facing the uncertainty of asset value, each investor chooses position so as to maximize her expected utility; that is, each investor faces the following utility maximizing problem:

where

$$
\max _{x} E[u(r)]
$$

$$
r(x)=\left\{\begin{array}{r}
V-p, \text { for } x=1 \\
0, \text { for } x=0 \\
p-V, \text { for } x=-1
\end{array}\right.
$$

\footnotetext{
${ }^{3}$ Chamley and Gale (1994), Neeman and Orosel (1997), Avery and Zemsky (1998), Lee (1998), Chari and Kehoe (2004), and Park and Sasbourian (2011) develop herding models that focus on information externality. Scharfstein and Stein (1990) and Dasgupta and Prat (2008) are models of herding among fund managers with reputation concern. Trueman (1994) and Ottaviani and Sorensen (2006) are models of herding among analyst forecasts. For a survey of herding models in various frameworks, see Bikchandani and Sharma (2001) and Hirshleifer and Teoh (2003). For empirical evidence of herding in security markets, see, for example, Lakonsishok et al. (1992), Christie and Huang (1995), Nofsinger and Sias (1999), Wermers (1999) and Sias (2004). For empirical evidence of herding among security analysts, see, for example, Graham (1999), Welch (2000) and Bernhardt et al (2006).
} 
Following Bell (1982), Loomes and Sugden (1982) and Quiggin (1994), we assume that investors are regret averse.

Assumption 1. Investors are regret averse with their utility functions take the following form:

$$
u(r)=r-f(\max \{r\}-r)
$$

where $\max \{r\} \equiv \max \{r(1), r(0), r(-1)\}$ and

$$
f(\max \{r\}-r)=\eta \sqrt{\max \{r\}-r}
$$

with $\eta>0$.

In our model, where there are three choices and two states, the best possible result is as follows:

$$
\max \{r\}=\left\{\begin{aligned}
1-p, & \text { if } V=1 \\
p, & \text { if } V=0
\end{aligned}\right.
$$

The function $f(\cdot)$ may be called "regret function" in the sense that when compared with the best possible result, there is always regret but no rejoice. We assume that $f(\cdot)$ is a square root function, where $\sqrt{r-\max \{r\}}$ measures the disutility of regret and $\eta>0$ reveals the magnitude of regret aversion. ${ }^{4}$

The basic framework of the market is similar to that in Glosten and Milgrom (1985). In a dealer market, a risky asset is traded at date $t=1,2, \cdots, T$. At date $T+1$, all positions are liquidated at the true value of the asset, whose prior distribution is $\operatorname{Pr}\{V=1\}=\operatorname{Pr}\{V=0\}=0.5$. Investors are a continuum of one, with a proportion of $\phi$ are informed traders and $1-\phi$ are liquidity traders. Each informed trader observes a conditionally independent signal $s \in\{0,1\}$ with $\operatorname{Pr}\{s=V \mid V\}=q>0.5$. Among informed traders, if the true value of the asset is $V=1$, a proportion of $\phi q$ will observe positive signal $s=1$, and the rest will observe negative signal $s=0$. If it is $V=0$, then, the proportion of investors with positive signals is $\phi(1-q)$, and the rest observe negative signals. ${ }^{5}$

At the beginning of the trading round at date $t$, a market maker sets price $p_{t}$ for both buy and sell orders. While each liquidity trader randomly submits a buy order or a sell order with equal probability, informed traders choose their positions basing on both public information and their private signals about asset value. From the pool of all investors' orders, the market maker randomly executes one piece of order at price $p_{t}$. Let $x_{t}$ denote the sign of the order executed at date $\mathrm{t}$ : if it

${ }^{4}$ In the present paper, regret is measured by $-f(\cdot)$ which is a convex function. In the original model of Bell $(1982,1983)$ and Loomes and Sugden $(1982,1987)$, regret is measured by a decreasingly concave function $f(\cdot)$, which is necessary to explain the paradox in Allais (1953) and experimental evidences in Kahneman and Tversky's (1979). As the present paper focuses on trading strategies in a sequential trading model, square root function is adopted to simplify the analysis. Nevertheless, important properties of the original model are still kept: there is a tradeoff between return and regret; regret is increasing in the difference between the best possible result and the actual result.

${ }^{5}$ If investors do not have private information and the price of the asset is fare in the sense that $p=E[V]$, then, investors' expected return is zero on any position. If so, investors will choose their positions only to avoid regret. However, we argue that such a setting is not realistic in the sense that profit is obviously the most important incentive for trading. Therefore, we assume there is asymmetric information among investors as in the model of Glosten and Milgrom (1985); this assumption enables us to analyze investors' decision making when they face the tradeoff between positive expected return and anticipated regret. 
is a buy order, then, $x_{t}=1$; if it is a sell order, then $x_{t}=0$. The history of previous trading is public information, which is denoted by $h_{t} \equiv\left\{\left(p_{\tau}, x_{\tau}\right), \tau=1,2, \cdots, t-1\right\}$. The public belief of asset value is defined as follows:

$$
\mu_{t} \equiv \operatorname{Pr}\left\{V=1 \mid h_{t}\right\}
$$

with $\mu_{1}=0.5$. The market maker is risk neutral who sets price equal to the expected value of the asset relative to public information:

$$
p_{t}=\mu_{t}
$$

After each round of trading, $x_{t}$ is announced to all market participants, and the public belief is updated through Bayesian learning: $\mu_{t+1}=\operatorname{Pr}\left\{V=1 \mid h_{t}, x_{t}\right\}{ }^{6}$

\section{Trading Strategy under Regret Aversion}

Let $E_{t}[\cdot]=E\left[\cdot \mid h_{t}\right]$ denote the expectation conditioning on public information, $E_{t}^{1}[\cdot]=$ $E\left[\cdot \mid h_{t}, s=1\right]$ the expectation conditioning on public information and positive signal $s=1$, and $E_{t}^{0}[\cdot]=E\left[\cdot \mid h_{t}, s=0\right]$ the expectation conditioning on public information and negative signal $s=0$. For informed traders, the expected value of the asset conditioning on their private signals is as follows:

$$
\begin{aligned}
& \mu_{t}^{1} \equiv E_{t}^{1}[V]=\frac{\mu_{t} q}{\mu_{t} q+\left(1-\mu_{t}\right)(1-q)} \\
& \mu_{t}^{0} \equiv E_{t}^{0}[V]=\frac{\mu_{t}(1-q)}{\mu_{t}(1-q)+\left(1-\mu_{t}\right) q}
\end{aligned}
$$

Because the price of the asset is set according to public information, an informed trader who takes a position according to her signal can earn a positive expected return; that is, $\mathrm{E}_{t}^{1}[r(1)]>0$ and $\mathrm{E}_{t}^{0}[r(0)]>0$. If investors do not regret, then, as described in Glosten and Milgrom (1985), informed investors always follow their private signals. As a result, order flow partially reveals investors' private information and public information about the asset accumulates overtime. In the limit, the price converges to the true value of asset; that is, as $t \rightarrow+\infty, p_{t} \rightarrow V$ almost surely. When investors have regret aversion, however, this result no more holds.

Table 1: Investment return.

\begin{tabular}{|r|l|l|l|l|l|}
\hline State & $\operatorname{Pr}\left\{\cdot \mid h_{t}\right\}$ & $\max \{r\}$ & $r(1)$ & $r(0)$ & $r(-1)$ \\
\hline$V=1$ & $\mu_{t}$ & $1-p_{t}$ & $1-p_{t}$ & 0 & $-\left(1-p_{t}\right)$ \\
\hline$V=0$ & $1-\mu_{t}$ & $p_{t}$ & $-p_{t}$ & 0 & $p_{t}$ \\
\hline
\end{tabular}

${ }^{6}$ In the original model of Glosten and Milgrom (1985), prices are set based on both public information and current order flow such that $p_{t}=E\left[V \mid h_{t}, x_{t}\right]$; that is, the price for buy orders is $p_{t}^{a}=E\left[V \mid h_{t}, x_{t}=1\right]$ while that for sell orders is $p_{t}^{b}=E\left[V \mid h_{t}, x_{t}=0\right]$. The bid-ask spread covers the market maker's loss in a trade with an informed trader. In order to focus on the effect of regret and simplify the analysis, the present paper assumes that there is no bid-ask spread. Neverthless, results of this paper can be extended to the case where bid-ask spread exists. 
Table 2: Conditional probability and investor's utility.

\begin{tabular}{|l|l|l|l|l|l|}
\hline State & $\operatorname{Pr}\left\{\cdot \mid h_{t}, s=1\right\}$ & $\operatorname{Pr}\left\{\cdot \mid h_{t}, s=0\right\}$ & $u(r(1))$ & $u(r(0))$ & $u(r(-1))$ \\
\hline$V=1$ & $\mu_{t}^{1}$ & $\mu_{t}^{0}$ & $1-p_{t}$ & $-\eta \sqrt{\left(1-p_{t}\right)}$ & $p_{t}-1-\eta \sqrt{2\left(1-p_{t}\right)}$ \\
\hline$V=0$ & $1-\mu_{t}^{1}$ & $1-\mu_{t}^{0}$ & $-p_{t}-\eta \sqrt{2 p_{t}}$ & $-\eta \sqrt{2 p_{t}}$ & $p_{t}$ \\
\hline
\end{tabular}

Consider informed traders' maximizing problems at date t. Table 1 shows the returns on different positions; Table 2 shows informed traders' utility, which not only contains the utility of investment return, but also reveals the disutility of regret.

Let $U_{t}^{S}(x) \equiv E_{t}^{S}[u(r(x))]$ denote an trader's expected utility who has signal $s$ and takes position $x$. Following Table 1 and Table 2, we have

$$
\begin{aligned}
& U_{t}^{S}(1)=\mu_{t}^{S}\left(1-p_{t}\right)-\left(1-\mu_{t}^{S}\right)\left(p_{t}+\eta \sqrt{2 p_{t}}\right) \\
& U_{t}^{S}(0)=-\eta \mu_{t}^{S} \sqrt{1-p_{t}}-\eta\left(1-\mu_{t}^{S}\right) \sqrt{p_{t}} \\
& U_{t}^{S}(-1)=\left(1-\mu_{t}^{S}\right) p_{t}-\mu_{t}^{S}\left(1-p_{t}+\eta \sqrt{2\left(1-p_{t}\right)}\right)
\end{aligned}
$$

Although the expected return is always positive if an informed trader follows her signal, she faces the tradeoff between expected return and expected disutility of regret. The optimal strategy for an informed trader with a signal $s$ is defined as bellows:

$$
x_{t}^{S}=\left\{\begin{array}{rc}
1, & \text { if } U_{t}^{S}(1) \geq \max \left\{U_{t}^{S}(0), U_{t}^{S}(-1)\right\} \\
0, & \text { if } U_{t}^{S}(0)>\max \left\{U_{t}^{S}(1), U_{t}^{S}(-1)\right\} \\
-1, & \text { otherwise }
\end{array}\right.
$$

To derive explicit expressions of $x_{t}^{1}$ and $x_{t}^{0}$, we first compare the expected utility on a long position with that on a short position.

Lemma 1 There exist $0<\mu<0.5<\bar{\mu}<1$ such that

$$
\begin{cases}U_{t}^{1}(1)<U_{t}^{1}(-\overline{1}) & \text { for } \mu_{t} \in(0, \underline{\mu}) \\ U_{t}^{1}(1)=U_{t}^{1}(-1) & \text { for } \mu_{t}=\underline{\mu} \\ U_{t}^{1}(1)>U_{t}^{1}(-1) & \text { for } \mu_{t} \in(\underline{\mu}, 1)\end{cases}
$$

holds for informed traders with signal $s=1$, and

$$
\begin{cases}U_{t}^{0}(1)<U_{t}^{0}(-1) & \text { for } \mu_{t} \in(0, \bar{\mu}) \\ U_{t}^{0}(1)=U_{t}^{0}(-1) & \text { for } \mu_{t}=\bar{\mu} \\ U_{t}^{0}(1)>U_{t}^{0}(-1) & \text { for } \mu_{t} \in(\bar{\mu}, 1)\end{cases}
$$

holds for informed traders with signal $s=0$.

Proof. Let $\lambda_{t} \equiv \frac{\mu_{t}}{1-\mu_{t}}, \lambda_{t}^{1} \equiv \frac{\mu_{t}^{1}}{1-\mu_{t}^{1}}, \lambda_{t}^{0} \equiv \frac{\mu_{t}^{0}}{1-\mu_{t}^{0}}$, and $\lambda_{q} \equiv \frac{q}{1-q}$ denote the likelihood ratios with respect to relative distributions. By the rule of Bayesian inference,

$$
\begin{aligned}
& \mu_{t}^{1}=\operatorname{Pr}\left\{\mathrm{V}=1 \mid h_{t}, s=1\right\}=\lambda_{t}^{1} \equiv \frac{\lambda_{t}^{1}}{1+\lambda_{t}^{1}}=\frac{\lambda_{t} \lambda_{q}}{1+\lambda_{t} \lambda_{q}} \\
& \mu_{t}^{0}=\operatorname{Pr}\left\{\mathrm{V}=1 \mid h_{t}, s=0\right\}=\lambda_{t}^{1} \equiv \frac{\lambda_{t}^{0}}{1+\lambda_{t}^{0}}=\frac{\lambda_{t} / \lambda_{q}}{1+\lambda_{t} / \lambda_{q}}
\end{aligned}
$$

Recall that $p_{t}=\mu_{t}$. For an informed trader with a positive signal $s=1$,

$$
U_{t}^{1}(1)-U_{t}^{1}(-1)=2\left(\mu_{t}^{1}-\mu_{t}\right)-\eta\left(1-\mu_{t}^{1}\right) \sqrt{2 \mu_{t}}+\eta \mu_{t}^{1} \sqrt{2\left(1-\mu_{t}\right)}
$$


Hence,

where

$$
U_{t}^{1}(1)-U_{t}^{1}(-1) \gtreqless 0 \Leftrightarrow M^{1}\left(\mu_{t}\right) \lesseqgtr \frac{\sqrt{2}}{\eta}
$$

$$
M^{1}\left(\mu_{t}\right) \equiv \frac{\left(1-\mu_{t}^{1}\right) \sqrt{\mu_{t}}-\mu_{t}^{1} \sqrt{\left(1-\mu_{t}\right)}}{\mu_{t}^{1}-\mu_{t}}=\frac{\sqrt{1+\frac{1}{\lambda_{t}}}-\lambda_{q} \sqrt{1+\lambda_{t}}}{\lambda_{q}-1}
$$

Because $\lambda_{t} \equiv \frac{\mu_{t}}{1-\mu_{t}}$ is strictly increasing in $\mu_{t}$ with $\left.\lambda_{t}\right|_{\mu_{t}=0.5}=1$, it is easy to see that $M^{1}\left(\mu_{t}\right)$ is strictly decreasing in $\mu_{t}$ with $M^{1}(0.5)=-1$ and $M^{1}\left(\mu_{t}\right) \rightarrow+\infty$ as $\mu_{t} \rightarrow 0$. By the continuity of $M^{1}\left(\mu_{t}\right)$, there is $\underline{\mu} \in\left(0, \frac{1}{2}\right)$ such that $M^{1}(\underline{\mu})=\frac{\sqrt{2}}{\eta}$. It is easy to see that $\underline{\mu}$ is the unique solution to $M^{1}\left(\mu_{t}\right)=\frac{\sqrt{2}}{\eta}$ and it does not depend on $t$.

For an informed trader with a negative signal, we have

$$
U_{t}^{0}(-1)-U_{t}^{0}(1)=2\left(\mu_{t}-\mu_{t}^{0}\right)-\eta \mu_{t}^{0} \sqrt{2\left(1-\mu_{t}\right)}+\eta\left(1-\mu_{t}^{0}\right) \sqrt{2 \mu_{t}}
$$

Therefore,

$$
U_{t}^{0}(-1)-U_{t}^{0}(1) \gtreqless 0 \Leftrightarrow M^{0}\left(\mu_{t}\right) \lesseqgtr \frac{\sqrt{2}}{\eta}
$$

where

$$
M^{0}\left(\mu_{t}\right) \equiv \frac{\mu_{t}^{0} \sqrt{\left(1-\mu_{t}\right)}-\left(1-\mu_{t}^{0}\right) \sqrt{\mu_{t}}}{\mu_{t}-\mu_{t}^{0}}=\frac{\sqrt{1+\lambda_{t}}-\lambda_{q} \sqrt{1+\frac{1}{\lambda_{t}}}}{\lambda_{q}-1}
$$

$M^{0}\left(\mu_{t}\right)$ is strictly increasing in $\mu_{t}$ with $M^{0}(0.5)=-1$ and $M^{0}\left(\mu_{t}\right) \rightarrow+\infty$ as $\mu_{t} \rightarrow 1$. Therefore, there is $\bar{\mu} \in\left(\frac{1}{2}, 1\right)$ such that $\left.M_{t}^{0}\right|_{\mu_{t}=\bar{\mu}}=\frac{\sqrt{2}}{\eta}$. It is easy to see that $\bar{\mu}$ is the unique solution to $M^{0}\left(\mu_{t}\right)=\frac{\sqrt{2}}{\eta}$ and it does not depend on $t$. As shown in Figure 1, above results imply the holding of Lemma 1 .

Q.E.D.

Lemma 1 shows that an informed trader may choose the position that is opposite to her signal. For example, for an informed trader who has a positive signal, the expected return on a long position is higher than that on a short position. However, if the public belief $\mu_{t}$ is low enough, then, the informed trader prefers to take a short position in order to avoid regret. For an informed trader with a negative signal, the situation is opposite: she will ignore her signal and take a long position if the public belief is high enough.

Next, we consider the choice of not trading; however, it is found that investors always choose trading no matter what value the public belief takes.

Lemma 2 For informed traders with signal $s=1$,

$$
\left\{\begin{array}{cl}
U_{t}^{1}(0)<U_{t}^{1}(-1) & \text { for } \mu_{t} \in(0, \underline{\mu}) \\
U_{t}^{1}(0)<U_{t}^{1}(1) & \text { for } \mu_{t} \in[\underline{\mu}, \overline{1})
\end{array}\right.
$$

for informed traders with signal $s=0$,

$$
\left\{\begin{array}{cl}
U_{t}^{0}(0)<U_{t}^{0}(-1) & \text { for } \mu_{t} \in(0, \bar{\mu}] \\
U_{t}^{0}(0)<U_{t}^{0}(1) & \text { for } \mu_{t} \in(\bar{\mu}, 1) .
\end{array}\right.
$$

Proof. For an informed trader with a positive signal, 


$$
\begin{aligned}
& U_{t}^{1}(1)-U_{t}^{1}(0)=\mu_{t}^{1}-\mu_{t}-\eta\left(1-\mu_{t}^{1}\right) \sqrt{2 \mu_{t}}+\eta \mu_{t}^{1} \sqrt{1-\mu_{t}}+\eta\left(1-\mu_{t}^{1}\right) \sqrt{\mu_{t}} \\
& U_{t}^{1}(0)-U_{t}^{1}(-1)=\mu_{t}^{1}-\mu_{t}+\eta \mu_{t}^{1} \sqrt{2\left(1-\mu_{t}\right)}-\eta \mu_{t}^{1} \sqrt{1-\mu_{t}}-\eta\left(1-\mu_{t}^{1}\right) \sqrt{\mu_{t}}
\end{aligned}
$$

Thus,

where

$$
\begin{aligned}
& U_{t}^{1}(1)-U_{t}^{1}(0) \gtreqless 0 \Leftrightarrow K^{1}\left(\mu_{t}\right) \lesseqgtr \frac{\sqrt{2}}{\eta} \\
& U_{t}^{1}(0)-U_{t}^{1}(-1) \gtreqless 0 \Leftrightarrow G^{1}\left(\mu_{t}\right) \lesseqgtr \frac{\sqrt{2}}{\eta}
\end{aligned}
$$

$$
\begin{aligned}
& K^{1}\left(\mu_{t}\right) \equiv \frac{(2-\sqrt{2})\left(1-\mu_{t}^{1}\right) \sqrt{\mu_{t}}-\sqrt{2} \mu_{t}^{1} \sqrt{1-\mu_{t}}}{\mu_{t}^{1}-\mu_{t}}=\frac{(2-\sqrt{2}) \sqrt{1+\frac{1}{\lambda_{t}}}-\sqrt{2} \lambda_{q} \sqrt{1+\lambda_{t}}}{\lambda_{q}-1} \\
& G^{1}\left(\mu_{t}\right) \equiv \frac{\sqrt{2}\left(1-\mu_{t}^{1}\right) \sqrt{\mu_{t}}-(2-\sqrt{2}) \mu_{t}^{1} \sqrt{1-\mu_{t}}}{\mu_{t}^{1}-\mu_{t}}=\frac{\sqrt{2} \sqrt{1+\frac{1}{\lambda_{t}}}-(2-\sqrt{2}) \lambda_{q} \sqrt{1+\lambda_{t}}}{\lambda_{q}-1}
\end{aligned}
$$

Both $K^{1}\left(\mu_{t}\right)$ and $G^{1}\left(\mu_{t}\right)$ are strictly decreasing in $\mu_{t}$. As $\mu_{t} \rightarrow 1, K^{1}\left(\mu_{t}\right) \rightarrow+\infty$ and $G^{1}\left(\mu_{t}\right) \rightarrow+\infty$; as $\mu_{t} \rightarrow 0, K^{1}\left(\mu_{t}\right) \rightarrow-\infty$ and $G^{1}\left(\mu_{t}\right) \rightarrow-\infty$. Hence, $K^{1}\left(\mu_{t}\right)=\frac{\sqrt{2}}{\eta}$ has a unique solution $\underline{\kappa}$ and $G^{1}\left(\mu_{t}\right)=\frac{\sqrt{2}}{\eta}$ has a unique solution $\underline{v}$. Furthermore, it is easy to see that for given value of $\mu_{t}, G^{1}\left(\mu_{t}\right)>M^{1}\left(\mu_{t}\right)>K^{1}\left(\mu_{t}\right)$ holds. As shown in Figure 1, this implies that $K^{1}\left(\mu_{t}\right)<\frac{\sqrt{2}}{\eta}$ and $U_{t}^{1}(1)>U_{t}^{1}(0)$ hold for $\mu_{t} \in[\underline{\mu}, 1)$, while $G^{1}\left(\mu_{t}\right)>\frac{\sqrt{2}}{\eta}$ and $U_{t}^{1}(-1)>U_{t}^{1}(0)$ hold for $\mu_{t} \in(0, \underline{\mu}]$.

For an informed trader with negative signal,

Hence,

$$
\begin{aligned}
& U_{t}^{0}(-1)-U_{t}^{0}(0)=\mu_{t}-\mu_{t}^{0}-\eta \mu_{t}^{0} \sqrt{2\left(1-\mu_{t}\right)}+\eta \mu_{t}^{0} \sqrt{1-\mu_{t}}+\eta\left(1-\mu_{t}^{0}\right) \sqrt{\mu_{t}} \\
& U_{t}^{0}(0)-U_{t}^{0}(1)=\mu_{t}-\mu_{t}^{0}+\eta\left(1-\mu_{t}^{0}\right) \sqrt{2 \mu_{t}}-\eta \mu_{t}^{0} \sqrt{1-\mu_{t}}-\eta\left(1-\mu_{t}^{0}\right) \sqrt{\mu_{t}}
\end{aligned}
$$

$$
\begin{aligned}
& U_{t}^{0}(-1)-U_{t}^{0}(0) \gtreqless 0 \Leftrightarrow K^{0}\left(\mu_{t}\right) \lesseqgtr \frac{\sqrt{2}}{\eta} \\
& U_{t}^{0}(0)-U_{t}^{0}(1) \gtreqless 0 \Leftrightarrow G^{0}\left(\mu_{t}\right) \lesseqgtr \frac{\sqrt{2}}{\eta}
\end{aligned}
$$

where

$$
\begin{aligned}
& K^{0}\left(\mu_{t}\right) \equiv \frac{(2-\sqrt{2}) \mu_{t}^{0} \sqrt{1-\mu_{t}}-\sqrt{2}\left(1-\mu_{t}^{0}\right) \sqrt{\mu_{t}}}{\mu_{t}-\mu_{t}^{0}}=\frac{(2-\sqrt{2}) \sqrt{1+\lambda_{t}}-\sqrt{2} \lambda_{q} \sqrt{1+\frac{1}{\lambda_{t}}}}{\lambda_{q}-1} \\
& G^{0}\left(\mu_{t}\right) \equiv \frac{\sqrt{2} \mu_{t}^{0} \sqrt{1-\mu_{t}}-(2-\sqrt{2})\left(1-\mu_{t}^{0}\right) \sqrt{\mu_{t}}}{\mu_{t}-\mu_{t}^{0}}=\frac{\sqrt{2} \sqrt{1+\lambda_{t}}-(2-\sqrt{2}) \lambda_{q} \sqrt{1+\frac{1}{\lambda_{t}}}}{\lambda_{q}-1}
\end{aligned}
$$

Both $K^{0}\left(\mu_{t}\right)$ and $G^{0}\left(\mu_{t}\right)$ are strictly increasing in $\mu_{t}$. As $\mu_{t} \rightarrow 1, K^{0}\left(\mu_{t}\right) \rightarrow-\infty$ and $G^{0}\left(\mu_{t}\right) \rightarrow-\infty$; as $\mu_{t} \rightarrow 0, K^{0}\left(\mu_{t}\right) \rightarrow+\infty$ and $G^{0}\left(\mu_{t}\right) \rightarrow+\infty$. Hence, $K^{0}\left(\mu_{t}\right)=\frac{\sqrt{2}}{\eta}$ has a unique solution $\bar{\kappa}$ and $G^{0}\left(\mu_{t}\right)=\frac{\sqrt{2}}{\eta}$ has a unique solution $\bar{v}$. Because $G^{0}\left(\mu_{t}\right)>M^{0}\left(\mu_{t}\right)>$ $K^{0}\left(\mu_{t}\right)$, as shown in Figure $1, \quad K^{0}\left(\mu_{t}\right)<\frac{\sqrt{2}}{\eta}$ and $U_{t}^{0}(-1)>U_{t}^{0}(0)$ hold for $\mu_{t} \in(0, \bar{\mu}]$, while $G^{0}\left(\mu_{t}\right)>\frac{\sqrt{2}}{\eta}$ and $U_{t}^{0}(1)>U_{t}^{0}(0)$, hold for $\mu_{t} \in[\bar{\mu}, 1)$. This completes the proof of Lemma 2 .

Q.E.D. 


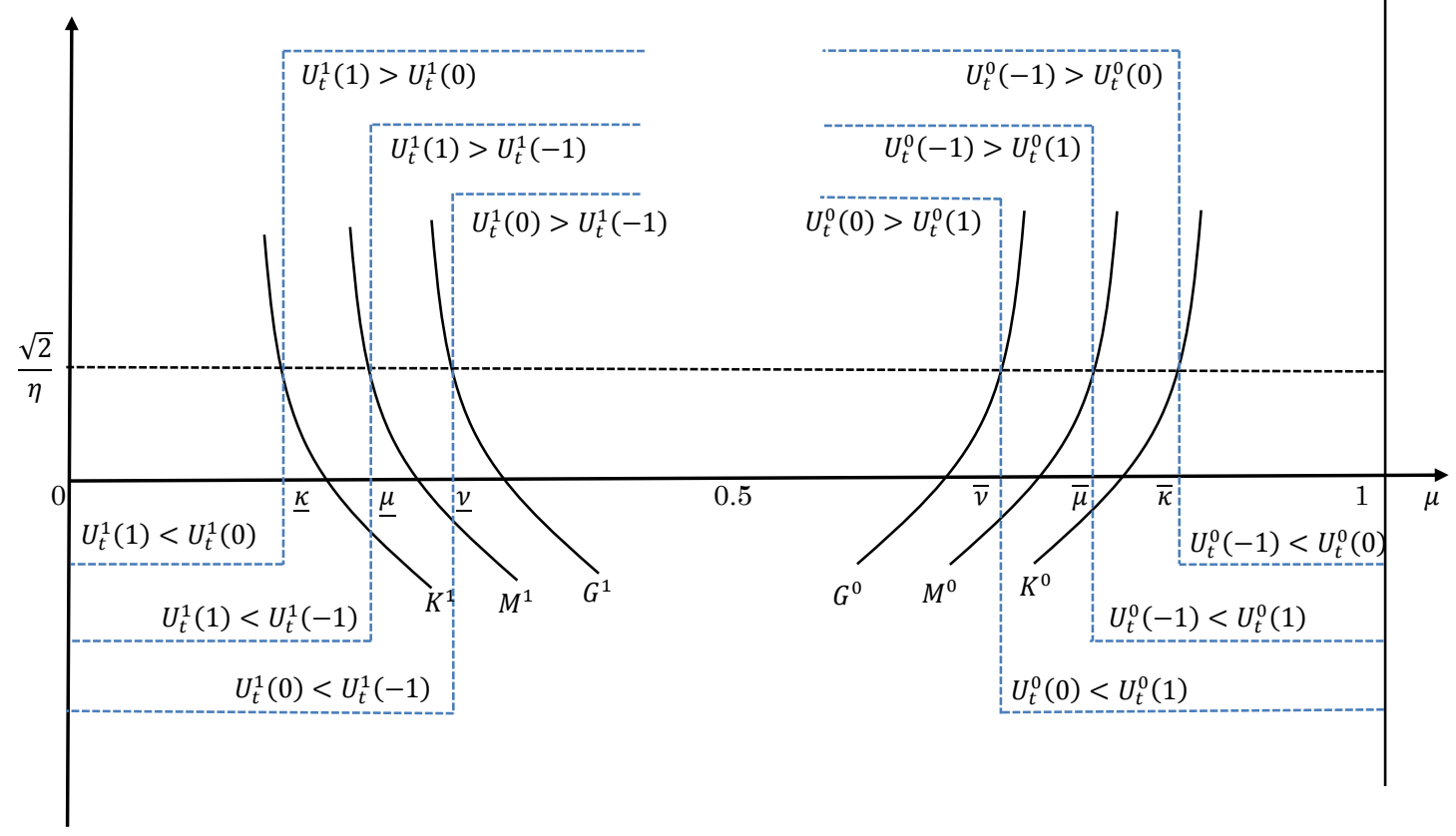

Figure 1

Figure 1 summarizes the results of Lemma 1 and Lemma 2. Following these results, we obtain informed traders' optimal trading strategies.

Proposition 1 Under Assumption 1, informed traders' optimal trading strategies for as follows:

$$
\begin{aligned}
& x_{t}^{1}=\left\{\begin{array}{cc}
-1 & \text { for } \mu_{t} \in(0, \underline{\mu}) \\
1 & \text { for } \mu_{t} \in[\mu, \overline{1}) ;
\end{array}\right. \\
& x_{t}^{0}=\left\{\begin{array}{cl}
-1 & \text { for } \mu_{t} \in(\overline{0}, \bar{\mu}) \\
1 & \text { for } \mu_{t} \in[\bar{\mu}, 1) .
\end{array}\right.
\end{aligned}
$$

An important implication of Proposition 1 is that informational cascade may occur in the market. By the definition of Bikhchandani et al. (1992), an informational cascade of buy occurs if it is optimal for all informed traders to submit buy orders; an informational cascade of sell occurs if it is optimal for all informed traders to submit sell orders. $\bar{\mu}$ and $\underline{\mu}$ in Proposition 1 are thresholds values for informational cascade in the present model: a buy cascade occurs if the public belief is higher than $\bar{\mu}$ and a sell cascade occurs if the public belief is lower than $\mu$. A formal statement about herding and information accumulation is given in the next section.

Finally, we examine how the magnitude of regret aversion and the precision of private information affect informed traders' optimal trading strategies.

Corollary $1 \bar{\mu}$ and $\underline{\mu}$ depend on $\eta$ and $q$ with

(i) $\frac{\partial \underline{\mu}}{\partial \eta}>0, \frac{\partial \bar{\mu}}{\partial \eta}<0$;

(ii) $\frac{\partial \underline{\mu}}{\partial q}<0, \frac{\partial \bar{\mu}}{\partial q}>0$. 
Proof. As shown in the proof of Lemma $1, \underline{\mu}$ is the solution to $M^{1}(\underline{\mu})=\frac{\sqrt{2}}{\eta}$. Hence, $\frac{\partial\left(M^{1}(\underline{\mu})-\frac{\sqrt{2}}{\eta}\right)}{\partial \eta}=\frac{\partial M^{1}}{\partial \mu} \frac{\partial \underline{\mu}}{\partial \eta}+\frac{\sqrt{2}}{\eta^{2}}=0$. Since $\frac{\partial M^{1}}{\partial \mu}<0$, we have $\frac{\partial \underline{\mu}}{\partial \eta}>0$. Similarly, $\bar{\mu}$ is the solution to $M^{0}(\underline{\mu})=\frac{\sqrt{2}}{\eta}$. Following the result that $\frac{\partial\left(M^{0}(\underline{\mu})-\frac{\sqrt{2}}{\eta}\right)}{\partial \eta}=\frac{\partial M^{0}}{\partial \mu} \frac{\partial \bar{\mu}}{\partial \eta}+\frac{\sqrt{2}}{\eta^{2}}=0$ and $\frac{\partial M^{0}}{\partial \mu}>0$, we obtain $\frac{\partial \bar{\mu}}{\partial \eta}<0$.

As the effect of $q, \frac{\partial \underline{\mu}}{\partial q}<0$ holds becasue $\frac{\partial\left(M^{1}(\underline{\mu})-\frac{\sqrt{2}}{\eta}\right)}{\partial q}=\frac{\partial M^{1}}{\partial \mu} \frac{\partial \underline{\mu}}{\partial q}+\frac{\partial M^{1}}{\partial \lambda_{q}} \frac{\partial \lambda_{q}}{\partial q}=0, \frac{\partial M^{1}}{\partial \mu}<0$, $\frac{\partial M^{1}}{\partial \lambda_{q}}<0$ and $\frac{\partial \lambda_{q}}{\partial q}>0$. Similarly, $\frac{\partial \bar{\mu}}{\partial q}>0$ holds becasue $\frac{\partial\left(M^{0}(\underline{\mu})-\frac{\sqrt{2}}{\eta}\right)}{\partial q}=\frac{\partial M^{0}}{\partial \mu} \frac{\partial \bar{\mu}}{\partial q}+\frac{\partial M^{0}}{\partial \lambda_{q}} \frac{\partial \lambda_{q}}{\partial q}=0$, $\frac{\partial M^{0}}{\partial \mu}>0, \frac{\partial M^{0}}{\partial \lambda_{q}}<0$ and $\frac{\partial \lambda_{q}}{\partial q}>0$.

Q.E.D.

\section{An Extended Model}

In this section, we analyze informed traders' trading strategies under a more general assumption about regret aversion.

Assumption 2. Investors are regret averse with their utility functions take the following form:

where $0 \leq z \leq 1$.

$$
u(r(x))=\left\{\begin{array}{cl}
r(x)-\eta \sqrt{\max \{r\}-r(x)} & \text { for } x=1,-1 \\
-z \eta \sqrt{\max \{r\}} & \text { for } x=0,
\end{array}\right.
$$

We obtain the following proposition under this assumption, which is the main result of the present paper.

Proposition 2 Under Assumption 2, informed traders' optimal trading strategies are as follows:

(i) In the case of $\sqrt{2}(1-q)<z<\frac{1}{\sqrt{2}}$, there exist $0<\underline{v}<\underline{\kappa}<0.5<\bar{\kappa}<\bar{v}<1$, such that

$$
\begin{aligned}
& x_{t}^{1}=\left\{\begin{array}{cc}
-1 & \text { for } \mu_{t} \in(0, \underline{v}] \\
0 & \text { for } \mu_{t} \in(\underline{v}, \underline{\kappa}) \\
1 & \text { for } \mu_{t} \in[\underline{\kappa}, 1)
\end{array}\right. \\
& x_{t}^{0}=\left\{\begin{array}{cc}
-1 & \text { for } \mu_{t} \in(0, \bar{\kappa}] \\
0 & \text { for } \mu_{t} \in(\bar{\kappa}, \bar{v}) \\
1 & \text { for } \mu_{t} \in[\bar{v}, 1)
\end{array}\right.
\end{aligned}
$$

(ii) In the case of $z>\frac{1}{\sqrt{2}}$, there exist $0<\underline{\mu}<0.5<\bar{\mu}<1$, such that

$$
\begin{aligned}
& x_{t}^{1}=\left\{\begin{array}{cc}
-1 & \text { for } \mu_{t} \in(0, \mu) \\
1 & \text { for } \mu_{t} \in[\mu, \overline{1})
\end{array}\right. \\
& x_{t}^{0}=\left\{\begin{array}{cc}
-1 & \text { for } \mu_{t} \in(\overline{0}, \bar{\mu}) \\
1 & \text { for } \mu_{t} \in[\bar{\mu}, 1)
\end{array}\right.
\end{aligned}
$$

Proof. For an informed trader with signal $s=1$, 


$$
\begin{aligned}
& U_{t}^{1}(1)-U_{t}^{1}(-1)=2\left(\mu_{t}^{1}-\mu_{t}\right)-\eta\left(1-\mu_{t}^{1}\right) \sqrt{2 \mu_{t}}+\eta \mu_{t}^{1} \sqrt{2\left(1-\mu_{t}\right)} \\
& U_{t}^{1}(1)-U_{t}^{1}(0)=\mu_{t}^{1}-\mu_{t}-\eta\left(1-\mu_{t}^{1}\right) \sqrt{2 \mu_{t}}+z \eta \mu_{t}^{1} \sqrt{1-\mu_{t}}+z \eta\left(1-\mu_{t}^{1}\right) \sqrt{\mu_{t}} \\
& U_{t}^{1}(0)-U_{t}^{1}(-1)=\mu_{t}^{1}-\mu_{t}+\eta \mu_{t}^{1} \sqrt{2\left(1-\mu_{t}\right)}-z \eta \mu_{t}^{1} \sqrt{1-\mu_{t}}-z \eta\left(1-\mu_{t}^{1}\right) \sqrt{\mu_{t}}
\end{aligned}
$$

Therefore,

where

$$
\begin{aligned}
& U_{t}^{1}(1)-U_{t}^{1}(-1) \gtreqless 0 \Leftrightarrow M_{t}^{1} \lesseqgtr \frac{\sqrt{2}}{\eta} \\
& U_{t}^{1}(1)-U_{t}^{1}(0) \gtreqless 0 \Leftrightarrow \widehat{K}_{t}^{1} \lesseqgtr \frac{\sqrt{2}}{\eta} \\
& U_{t}^{1}(0)-U_{t}^{1}(-1) \gtreqless 0 \Leftrightarrow \widehat{G}_{t}^{1} \lesseqgtr \frac{\sqrt{2}}{\eta}
\end{aligned}
$$

$$
\begin{aligned}
& M^{1}\left(\mu_{t}\right)=\frac{\left(1-\mu_{t}^{1}\right) \sqrt{\mu_{t}}-\mu_{t}^{1} \sqrt{\left(1-\mu_{t}\right)}}{\mu_{t}^{1}-\mu_{t}}=\frac{\sqrt{1+\frac{1}{\lambda_{t}}}-\lambda_{q} \sqrt{1+\lambda_{t}}}{\lambda_{q}-1} \\
& \widehat{K}^{1}\left(\mu_{t}\right) \equiv \frac{(2-\sqrt{2} z)\left(1-\mu_{t}^{1}\right) \sqrt{\mu_{t}}-\sqrt{2} z \mu_{t}^{1} \sqrt{1-\mu_{t}}}{\mu_{t}^{1}-\mu_{t}}=\frac{(2-\sqrt{2} z) \sqrt{1+\frac{1}{\lambda_{t}}}-\sqrt{2} z \lambda_{q} \sqrt{1+\lambda_{t}}}{\lambda_{q}-1} \\
& \widehat{G}^{1}\left(\mu_{t}\right) \equiv \frac{z \sqrt{2}\left(1-\mu_{t}^{1}\right) \sqrt{\mu_{t}}-(2-\sqrt{2} z) \mu_{t}^{1} \sqrt{1-\mu_{t}}}{\mu_{t}^{1}-\mu_{t}}=\frac{\sqrt{2} z \sqrt{1+\frac{1}{\lambda_{t}}}-(2-\sqrt{2} z) \lambda_{q} \sqrt{1+\lambda_{t}}}{\lambda_{q}-1}
\end{aligned}
$$

$M^{1}\left(\mu_{t}\right), \widehat{K}^{1}\left(\mu_{t}\right)$ and $\widehat{K}^{1}\left(\mu_{t}\right)$ are strictly decreasing in $\mu_{t}$. Let $\mu$ be the unique solution to $M_{t}^{1}=\frac{\sqrt{2}}{\eta}, \underline{\kappa}$ the unique solution to $\widehat{K}^{1}\left(\mu_{t}\right)=\frac{\sqrt{2}}{\eta}$, and $\underline{v}$ the unique solution to $\widehat{G}^{1}\left(\mu_{t}\right)=\frac{\sqrt{2}}{\eta}$. It is easy to see that $\underline{\mu}, \underline{\kappa}$ and $\underline{v}$ are well defined. Note that $\widehat{K}^{1}\left(\mu_{t}\right)>M^{1}\left(\mu_{t}\right)>\widehat{G}^{1}\left(\mu_{t}\right)$ holds for $z<\frac{1}{\sqrt{2}}$; furthermore, $\widehat{K}^{1}(0.5)=\frac{2 \sqrt{2}-\frac{2 z}{1-q}}{\lambda_{q}-1}<0$ holds when $\mathrm{z}>\sqrt{2}(1-q)$. Therefore, as shown in Figure 2, $\quad 0<\underline{v}<\underline{\mu}<\underline{\kappa}<0.5$.

For an informed trader with signal $s=0$,

$$
\begin{aligned}
& U_{t}^{0}(-1)-U_{t}^{0}(1)=2\left(\mu_{t}^{0}-\mu_{t}\right)-\eta \mu_{t}^{0} \sqrt{2\left(1-\mu_{t}\right)}+\eta\left(1-\mu_{t}^{0}\right) \sqrt{2 \mu_{t}} \\
& U_{t}^{0}(-1)-U_{t}^{0}(0)=\mu_{t}^{0}-\mu_{t}-\eta \mu_{t}^{0} \sqrt{2\left(1-\mu_{t}\right)}+z \eta \mu_{t}^{0} \sqrt{1-\mu_{t}}+z \eta\left(1-\mu_{t}^{0}\right) \sqrt{\mu_{t}}(59) \\
& U_{t}^{0}(0)-U_{t}^{0}(1)=\mu_{t}^{0}-\mu_{t}+\eta\left(1-\mu_{t}^{0}\right) \sqrt{2 \mu_{t}}-z \eta \mu_{t}^{0} \sqrt{1-\mu_{t}}-z \eta\left(1-\mu_{t}^{0}\right) \sqrt{\mu_{t}}
\end{aligned}
$$

Hence,

$$
\begin{aligned}
& U_{t}^{0}(-1)-U_{t}^{0}(1) \gtreqless 0 \Leftrightarrow M^{0}\left(\mu_{t}\right) \lesseqgtr \frac{\sqrt{2}}{\eta} \\
& U_{t}^{0}(-1)-U_{t}^{0}(0) \gtreqless 0 \Leftrightarrow \widehat{K}^{0}\left(\mu_{t}\right) \lesseqgtr \frac{\sqrt{2}}{\eta} \\
& U_{t}^{0}(0)-U_{t}^{0}(1) \gtreqless 0 \Leftrightarrow \widehat{G}^{0}\left(\mu_{t}\right) \lesseqgtr \frac{\sqrt{2}}{\eta}
\end{aligned}
$$

where

$$
\begin{aligned}
& M^{0}\left(\mu_{t}\right) \equiv \frac{\mu_{t}^{0} \sqrt{\left(1-\mu_{t}\right)}-\left(1-\mu_{t}^{0}\right) \sqrt{\mu_{t}}}{\mu_{t}-\mu_{t}^{0}}=\frac{\sqrt{1+\lambda_{t}}-\lambda_{q} \sqrt{1+\frac{1}{\lambda_{t}}}}{\lambda_{q}-1} \\
& \widehat{K}^{0}\left(\mu_{t}\right) \equiv \frac{(2-\sqrt{2} z) \mu_{t}^{0} \sqrt{1-\mu_{t}}-\sqrt{2} z\left(1-\mu_{t}^{0}\right) \sqrt{\mu_{t}}}{\mu_{t}-\mu_{t}^{0}}=\frac{(2-\sqrt{2} z) \sqrt{1+\lambda_{t}}-\sqrt{2} z \lambda_{q} \sqrt{1+\frac{1}{\lambda_{t}}}}{\lambda_{q}-1} \\
& \widehat{G}^{0}\left(\mu_{t}\right) \equiv \frac{z \sqrt{2} \mu_{t}^{0} \sqrt{1-\mu_{t}}-(2-\sqrt{2} z)\left(1-\mu_{t}^{0}\right) \sqrt{\mu_{t}}}{\mu_{t}-\mu_{t}^{0}}=\frac{\sqrt{2} z \sqrt{1+\lambda_{t}}-(2-\sqrt{2} z) \lambda_{q} \sqrt{1+\frac{1}{\lambda_{t}}}}{\lambda_{q}-1}
\end{aligned}
$$

$M^{0}\left(\mu_{t}\right), \widehat{K}^{0}\left(\mu_{t}\right)$ and $\widehat{K}^{0}\left(\mu_{t}\right)$ are strictly increasing in $\mu_{t}$. Let $\bar{\mu}$ be the unique solution to 
$M_{t}^{1}=\frac{\sqrt{2}}{\eta}, \bar{\kappa}$ the unique solution to $\widehat{K}^{0}\left(\mu_{t}\right)=\frac{\sqrt{2}}{\eta}$, and $\bar{v}$ the unique solution to $\hat{G}^{0}\left(\mu_{t}\right)=\frac{\sqrt{2}}{\eta}$. It is easy to see that $\bar{\mu}, \bar{\kappa}$ and $\bar{v}$ are well defined. Note that $\widehat{K}^{0}\left(\mu_{t}\right)>M^{0}\left(\mu_{t}\right)>\widehat{G}^{0}\left(\mu_{t}\right)$ holds for $z<\frac{1}{\sqrt{2}}$; furthermore, $\widehat{K}^{0}(0.5)=\frac{2 \sqrt{2}-\frac{2 z}{1-q}}{\lambda_{q}-1}<0$ holds when $\mathrm{z}>\sqrt{2}(1-q)$. Therefore, as shown in Figure 2, we have $0.5<\bar{\kappa}<\bar{\mu}<\bar{v}<1$. Therefore, statement (i) of Proposition 2 holds.

In the case of $z>\frac{1}{\sqrt{2}}, \widehat{G}^{1}\left(\mu_{t}\right)>M^{1}\left(\mu_{t}\right)>\widehat{K}^{1}\left(\mu_{t}\right)$ and $M^{1}(0.5)<0$ hold for investors with positive signal; $\hat{G}^{0}\left(\mu_{t}\right)>M^{0}\left(\mu_{t}\right)>\widehat{K}^{0}\left(\mu_{t}\right)$ and $M^{0}(0.5)<0$ hold for investors with negative signal. Therefore, similar to the situation shown in Figure 1, statement (ii) of Proposition 2 holds.

Q.E.D.

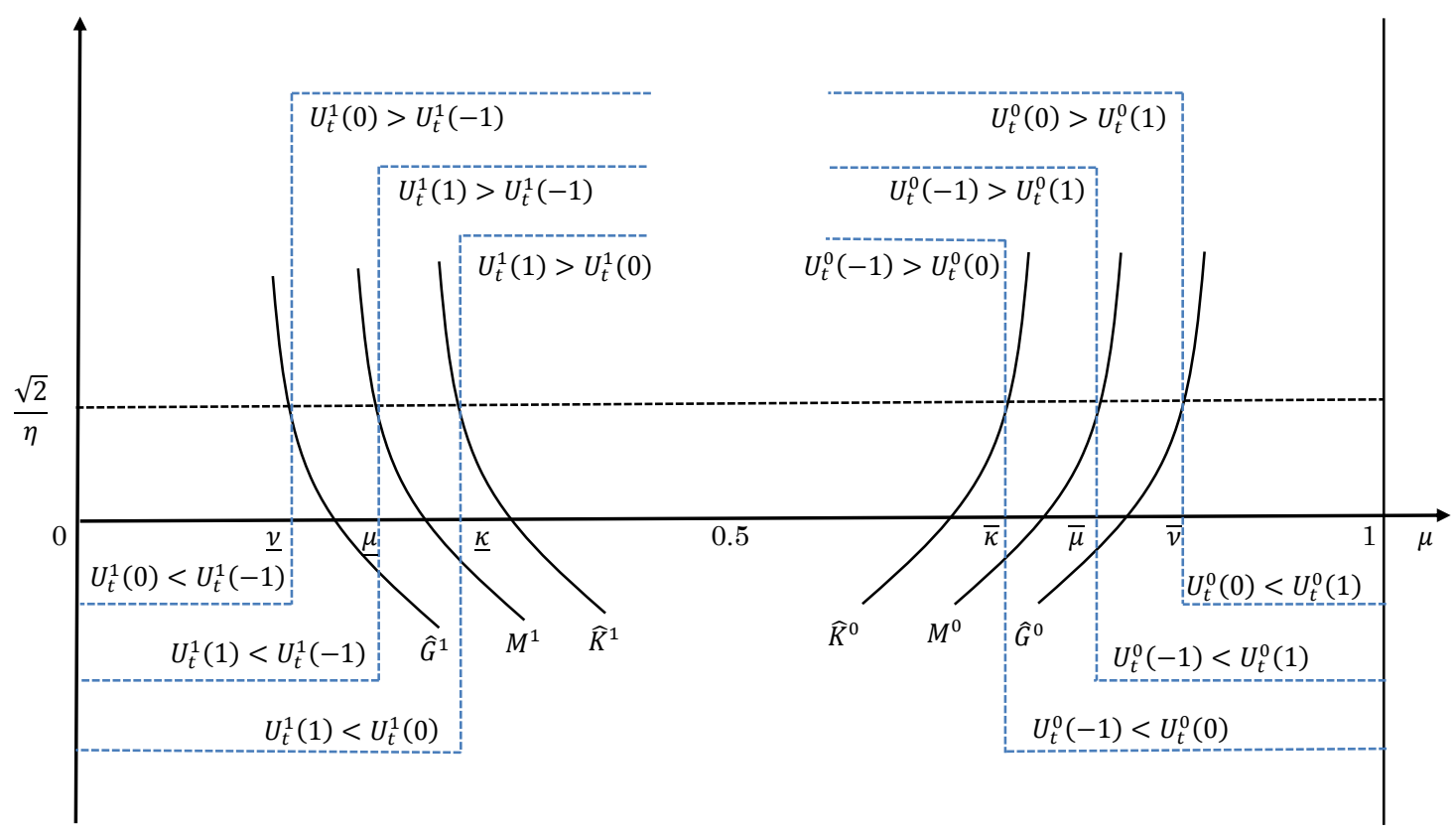

Figure 2

Statement (i) examines the case where investors' magnitude of regret aversion is relatively small when they do not trade. It shows that when market price takes moderate values, investors take positions according to their private information. When asset price rises above a certain level, then, while investors with positive signals still buy, those with negative signals will choose not to trade; if price becomes even higher, than all investors will choose to buy in spite of their private information. If asset price drops below a certain value, investors with positive signals first choose to stay aside; if the price drops even more deeply, they will re-enter the market and join the sellers.

Statement (ii) analyzes the case where investors' magnitude of regret aversion is relatively strong even when they do not trade. Optimal strategies in this case are the same as those described in Proposition 1.

Corollary 2 and Corollary 3 below describe investors' herding behavior and information accumulation in the market. As these corollaries are direct implications of Proposition 2, proofs for them are abbreviated. 
Corollary 2 In case $\sqrt{2}(1-q)<z<\frac{1}{\sqrt{2}}$, trading and information accumulation in the market are as follows.

(i) If $\mu_{t} \in[\underline{v}, \bar{v}]$, then, there is no cascade in the market: informed traders take positions following their private signals, and the public belief evolves after trading. That is,

$$
\begin{aligned}
& x_{t}^{1}=1, x_{t}^{0}=-1 \\
& \mu_{t+1}= \begin{cases}\frac{\mu_{t} \delta}{\mu_{t} \delta+\left(1-\mu_{t}\right)(1-\delta)} & \text { if } x_{t}=1 \\
\frac{\mu_{t}(1-\delta)}{\mu_{t}(1-\delta)+\left(1-\mu_{t}\right) \delta} & \text { if } x_{t}=-1\end{cases}
\end{aligned}
$$

(ii) If $\mu_{t} \in(\bar{v}, \bar{\kappa})$, then, an partial buy-cascade occurs: informed traders with positive signals submit to buy, but those with negative signals do not trade; information accumulation in the market slows down. That is,

$$
\begin{aligned}
& x_{t}^{1}=1, \quad x_{t}^{0}=0 \\
& \mu_{t+1}= \begin{cases}\frac{\mu_{t} \xi}{\mu_{t} \xi+\left(1-\mu_{t}\right)(1-\zeta)} & \text { if } x_{t}=1 \\
\frac{\mu_{t}(1-\xi)}{\mu_{t}(1-\xi)+\left(1-\mu_{t}\right) \zeta} & \text { if } x_{t}=-1\end{cases}
\end{aligned}
$$

where $\xi=\frac{\phi q+(1-\phi) / 2}{1-\phi(1-q)}$ and $\zeta=\frac{(1-\phi) / 2}{1-\phi q}$

(iii) If $\mu_{t} \in(\underline{\kappa}, \underline{v})$, then, an partial sell-cascade occurs: informed traders with negative signals submit to sell but those with positive signals do not trade; information accumulation in the market slows down. That is,

$$
\begin{aligned}
& x_{t}^{1}=0, x_{t}^{0}=-1 \\
& \mu_{t+1}= \begin{cases}\frac{\mu_{t} \zeta}{\mu_{t} \zeta+\left(1-\mu_{t}\right)(1-\xi)} & \text { if } x_{t}=1 \\
\frac{\mu_{t}(1-\zeta)}{\mu_{t}(1-\zeta)+\left(1-\mu_{t}\right) \xi} & \text { if } x_{t}=-1\end{cases}
\end{aligned}
$$

(iv) If $\mu_{t} \in[\bar{\kappa}, 1)$ then, a buy-cascade occurs: all informed traders submit buy orders and information accumulation in the market stops:

$$
\begin{aligned}
& x_{t}^{1}=x_{t}^{0}=1 \\
& \mu_{t+1}=\mu_{t}
\end{aligned}
$$

(v) If $\mu_{t} \in(0, \underline{\kappa}]$, then, a sell-cascade occurs: all informed traders submit sell orders and information accumulation in the market stops:

$$
\begin{aligned}
& x_{t}^{1}=x_{t}^{0}=-1 \\
& \mu_{t+1}=\mu_{t}
\end{aligned}
$$

Corollary 2 focuses on the case where investors' magnitude of regret aversion is relatively small when they do not trade. It is shown that when the price is very low or very high, a "partial cascade" occurs where some informed traders ignore their private information and do not trade. If the price takes even more extreme values, a partial cascade will develop into a full-scale cascade. The reason for investors to join a partial cascade or a full-scale cascade is that they do not want to bet against the market and regret afterwards.

Corollary 3 In case $z>\frac{1}{\sqrt{2}}$, trading and information accumulation in the market are as follows.

(i) If $\mu_{t} \in[\mu, \bar{\mu}]$, there is no cascade in the market: informed traders take positions following their private signals, and the public belief evolves after trading. That is,

$$
x_{t}^{1}=1, x_{t}^{0}=-1
$$




$$
\mu_{t+1}= \begin{cases}\frac{\mu_{t} \delta}{\mu_{t} \delta+\left(1-\mu_{t}\right)(1-\delta)} & \text { if } x_{t}=1 \\ \frac{\mu_{t}(1-\delta)}{\mu_{t}(1-\delta)+\left(1-\mu_{t}\right) \delta} & \text { if } x_{t}=-1\end{cases}
$$

(ii) If $\mu_{t}>\bar{\mu}$, then, an buy-cascade occurs: all informed traders submit buy orders and information accumulation in the market stops. That is,

$$
\begin{aligned}
& x_{t}^{1}=x_{t}^{0}=1 \\
& \mu_{t+1}=\mu_{t} .
\end{aligned}
$$

(iii) If $\mu_{t}<\mu$, then, an sell-cascade occurs: all informed traders submit sell orders and information accumulation in the market stops. That is,

$$
\begin{aligned}
& x_{t}^{1}=x_{t}^{0}=-1 \\
& \mu_{t+1}=\mu_{t} .
\end{aligned}
$$

Corollary 3 focus on the case that investors' magnitude of regret aversion is relatively strong even when they do not trade. In this case, the effect of regret is so strong that full scale cascades occur directly without the occurrence of partial cascades. Corollary 3 generalizes the result of Proposition 1.

\section{Conclusion}

The present paper examines the effect of regret on investment in a market where investors can choose to trade, or not to trade, an asset with uncertain value. Investors face the tradeoff between the expect utility of investment return and the expected disutility of regret. When the market price takes moderate values, investors take positions according to their private information. If the market price becomes very high or very low, some investors will ignore their own information and do not trade. In case the price is extremely high or extremely low, regret has such a strong effect that cascade occurs in the market.

\section{Reference}

[1] Avery, C. and P. Zemsky, 1998. Multidimensional uncertainty and herd behavior in financial markets. American Economic Review 88, 724-748.

[2] Bell, D., 1982. Regret in decision making under uncertainty. Operations Research 30, 961-981.

[3] Bell, D., 1983. Risk premiums for decision regret. Management Science 29, 1156-1166.

[4] Bell, D., 1985. Disappointment in decision making under uncertainty. Operations Research 33, $1-27$.

[5] Bernhardt, D., M. Campello, and E. Kutsoati, 2006. Who herds? Journal of Financial Economics 80, 657-675.

[6] Bikhchandani, S., D. Hirshleifer, and I. Welch, 1992. A theory of fads, fashion, custom, and cultural changes as informational cascades. Journal of Political Economy 100, 992-1026.

[7] Bikhchandani, S. and S. Sharma, 2001. Herd behavior in financial markets. IMF Staff Papers 47,3 .

[8] Camille, N., G. Coricelli, J. Sallet, P. Pradate-Diehl, J. Duhamel, and A. Sirigu, 2004. The involvement of the orbitofrontal cortex in the experience of regret. Science 304, 1167-1170.

[9] Chamley, C. and D. Gale, 1994. Information revelation and strategic delay in model of 
investment. Econometrica 62, 128-150.

[10] Chari, V. and P. Kehoe, 2004. Financial crises as herds: overturning the critiques. Journal of Economic Theory 119, 128-150.

[11] Coricelli, G., H. Critchley, M. Joffily, J. O’Doherty, A. Sirigu, and R. Dolan, 2005. Regret and its avoidance: a neuroimaging study of choice behavior. Nature Neuroscience 8, 1255-1262.

[12] Dasgupta, A. and A. Prat, 2008. Information aggregation in financial markets with career concerns. Journal of Economic Theory 143, 83-113.

[13] Dodonova, A., 2005. Applications of regret theory to asset pricing. Unpublished working paper, University of Ottawa.

[14] Fogel, S. and T. Berry, 2006. The disposition effect and individual investor decisions: the roles of regret and counterfactual alternatives. The Journal of Behavioral Finance 7, 107-116.

[15] Gilovich, T. and V. H. Medvec, 1995. The experience of regret: what, when, and why. Psychological Review 102, 379-395.

[16] Glosten, L. and P. Milgrom, 1985. Bid, ask and transaction prices in a specialist market with heterogeneously informed traders. Journal of Financial Economics 14, 71-100.

[17] Hirshleifer, D. and S. H. Teoh, 2003. Herd behaviour and cascading in capital markets: a review and synthesis. European Financial Management 9, 25-66.

[18] Lakonishok, J., A. Shleifer and R. Vishny, 1992, The impact of institutional trading on stock prices. Journal of Financial Economics 32, 23-43.

[19] Loomes, G. and R. Sugden, 1982. Regret theory: an alternative theory of rational choice under uncertainty. The Economic Journal 92, 805-824.

[20] Loomes, G. and R. Sugden, 1986. Disappointment and dynamic consistency in choice under uncertainty. Review of Economic Studies 53, 271-282.

[21] Loomes, G. and R. Sugden, 1987. Some implications of a more general form of regret theory. Journal of Economic Theory 41, 270-287.

[22] Lee, I. H., 1998. Market crashes and informational avalanches. Review of Economic Studies 65, 395-411.

[23] Loomes, G. and R. Sugden, 1982. Regret theory: an alternative theory of rational choice under uncertainty. The Economic Journal 92, 802-824.

[24] Michenaud, S. and B. Solnik, 2008. Applying regret theory to investment choices: currency hedging decisions. Journal of International Money and Finance 27, 677-694.

[25] Nofsinger, J. and R. Sias, 1999. Herding and feedback frading by institutional and individual investors. Journal of Finance 54, 2263-2295.

[26] Neeman, Z. and G. Orosel, 1997. Herding and the winner's curse in markets with sequential bids. Journal of Economic Theory 85, 91-121.

[27] Ottaviani, M. and P. Sorensen, 2006. Professional advice. Journal of Economic Theory 126, 120-142.

[28] Park, A. and H. Sabourian, 2011, Herd behavior in efficient Financial Markets. Econometric 79, 973-1026.

[29] Sias, R., 2004. Institutional Herding. Review of Financial Studies 17, 165-206.

[30] Scharfstein, S. and J. Stein, 1990. Herd behavior and investment. American Economic Review 80, 465-479.

[31] Trueman, B., 1994. Analyst forecasts and herding behavior. Review of Financial Study 7, 97-124.

[32] Welch, I., 2000. Herding among security analysts. Journal of Financial Economics 58, 369-396.

[33] Zeelenberg, M., W. van Dijk, J. van der Pligt, A. Manstead, P. van Empelen, and D. Reinderman, 1998. Emotional reactions to the outcomes of decisions: the role of 
counterfactural thought in the experience of regret and disappointment. Organizational Behavior and Human decision Processes 75, 117-141.

[34] Zeelenberg, M., 1999. Anticipated regret, expected feedback and behavioral decision making. Journal of Behavioral Decision Making 12: 93-106.

[35] Zeelenberg, M., W. van Dijk, A. Manstead, and J. van der Pligt, 2000. On gad decisions and disconfirmed expectancies: the psychology of regret and disappointment. Cognition and Emotion 14: 521-541. 